\title{
论 文 大飞机导航与控制专辑
}

\section{一种基于自适应神经网络的航空发动机故障诊断 方法}

\section{艾剑良 ${ }^{1}$, 杨䀬中 ${ }^{1,2 *}$}

\author{
1. 复旦大学航空航天系, 上海 200433; \\ 2. 中国航空无线电电子研究所, 上海 200233 \\ * E-mail: aijl@fudan.edu.cn
}

收稿日期：2017-07-13; 接受日期：2017-08-28; 网络发表日期：2017-12-20

上海市商用航空发动机领域联合创新计划(编号: AR908.D1RW.002)资助项目

摘要 航空发动机在使用过程中, 气路部件的性能不可避免地发生了蜕化, 相应的故障诊断技术对发动机的健康 管理系统具有重要意义. 本文针对发动机在设计点的非线性部件级模型, 借助PCC(Pearson correlation coefficient) 相关分析方法, 对神经网络的输入参数和输出参数的选取方式进行了优化. 以前馈型神经网络为基础, 针对常规 BP(back propagation)神经网络收玫速度不稳定、且容易陷入极小值的缺陷, 设计了一种新的自适应神经网络, 准 确估计了发动机部件的蜕化情况. 这种算法融合了比例因子和动量因子, 改善了网络的学习速率, 提高了神经网 络置信度和对发动机模型参数的泛化能力. 结果表明, 本文设计的自适应神经网络的精度优于常规BP神经网络, 并且在训练样本数较少时, 依然能够通过训练得到理想的网络, 保证发动机健康参数的故障检测具有较高精度.

关键词 航空发动机, 健康参数, 故障诊断, 自适应神经网络, 相关分析

\section{1 引言}

航空发动机在正常使用过程中，存在材料缺陷和 制造公差，并且长期反复经历高温高压、高速旋转和 振动等工作环境后，产生了疲劳和老化的积累，发动 机部件的实际工作状态逐渐偏离额定工作状态，出现 一定程度的故障. 其中, 由于气路性能故障在发动机 的三大主要故障(性能故障、结构强度故障和附件系 统故障)中的比例超过了 $90 \%{ }^{[1]}$ ，因而成为发动机故障 相关研究的主要内容. 气路性能的蜕化故障遍布于发 动机各个部分和整个使用周期，工程上通常选用一组
有限的参数来描述这些变化, 称之为健康参数. 发动 机健康参数的确定有助于提高机载模型的精度, 也是 研究发动机健康管理、实现综合控制等技术的基础. 机载传感器无法直接测量健康参数, 因此, 对健康参数 的估计是航空发动机故障检测和控制技术研究中的一 个热门方向.

航空发动机针对健康参数发生蜕化的故障诊断, 主要是根据一些传感器可测的信号(部件截面的压 力、温度、转子转速等), 对另一些未知信号(部件健 康参数中的效率系数、流量系数等) 的大小做出估计. 常用的状态估计方法有最小二乘估计、主成分估计、

引用格式: 艾剑良, 杨曦中. 一种基于自适应神经网络的航空发动机故障诊断方法. 中国科学: 技术科学, 2018, 48: 326-335

Ai J L, Yang X Z. Fault diagnosis of aero-engine based on self-adaptive neural network (in Chinese). Sci Sin Tech, 2018, 48: 326-335, doi: 10.1360/ N092017-00224 
最小均方差估计、最大似然参数估计、贝叶斯估计、 基于神经网络的参数估计等方法，其中各种故障诊断 方法又有很多变种和扩展方法. 基于神经网络的参数 估计方法是其中一种主要方法. 本文研究了利用自适 应神经网络估计发动机部件级模型在设计点的健康参 数的方法, 完成对发动机气路性能的故障诊断.

\section{2 航空发动机模型及其健康参数}

\section{1 航空发动机的部件级模型}

本文的研究对象为民用航空浴扇发动机. 根据发 动机的构造和功能, 主要可以划分为进气道、风扇、 压气机、燃烧室、浴轮和排气口等几个部分. 在双轴 浴扇发动机中，可以把风扇和压气机看作低压压气机 和高压压气机, 浴轮也分为高压浴轮和低压浴轮两部 分. 如果能够解析地描述这些部件的性能，那么就可 以精确描述整个发动机的工况.

在发动机部件特性已知的基础上，依次用非线性 方程描述各个部件工作时所遵守的气动热力学定律, 可以建立航空发动机设计点的部件级模型. 航空发动 机的“设计点”，指的是在给定的工作条件下(通常是飞 行高度、马赫数、大气压力、温度和湿度等), 选定工 作性能参数(例如: 压气机增压比、风扇增压比、涵道 比、健康参数等), 根据气动热力学过程推算发动机的 工作状态参数(主要为部件温度、压力、流量、整机 推力、耗油率等)。一般选取的设计点工作条件都是典 型的飞行状态，例如：地面慢车、最大起飞、空中慢 车、高空巡航、进近着陆等, 这些都是飞机飞行中的 关键过程, 准确了解这些在设计点的发动机特性, 对 于飞机和发动机的设计和安全工作至关重要.

部件级模型的优点在于精度相对较高，并且可以 详细计算发动机各个工作截面的参数. 航空发动机部 件级建模方法已经较为成熟，整套部件级模型求解过 程繁琐，具体参数和计算过程可以参阅文献[2 4]. 在 发动机的研制和使用过程中, 常常需要正确指定和区 分发动机轴向上的一些重要位置, 如各个部件相邻的 位置, 称之为主要截面, 为了方便使用, 将这些截面进 行编号. 本文所用的发动机数值模型各主要截面编号 情况如图1所示.

\section{2 航空发动机的健康参数}

故障诊断是一项跨学科的综合性技术. 航空发动

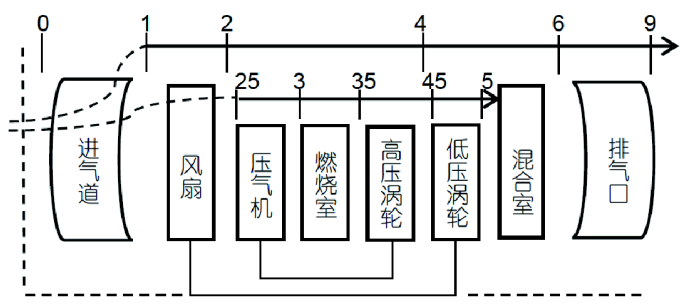

图 1 发动机部件级模型及其编号示意图

机的故障诊断，主要检测发动机的运行状态，诊断发 动机系统出现的故障情况、确定故障部位和大小, 以 便进一步采取调整控制律、部件维护或更换设备等合 理的解决方案. 发动机在使用过程中包含 4 种状态, 即 正常状态、健康蜕化状态、危险状态和失效状态. 而 发动机经常处于蜕化的过程, 如果能够在工作时检测 到性能蜕化的状态指标——健康参数, 就可以采取针 对性措施.

发动机健康参数主要包含效率系数和流量系数. 以效率系数 $\eta$ 为例, 它描述部件的真实效率 $\eta^{*}$ 与额定效 率 $\eta^{0}$ 的比值:

$\eta=\frac{\eta^{*}}{\eta^{0}}$

当部件未发生蜕化时, 发动机真实工况与额定工 况相同, $\eta^{*}=\eta^{0}$, 此时健康参数系数为 1 , 即 $\eta=1$.

研究中发现, 某型号发动机的主要部件发生均蜕 化 $2 \%$ 后, 发动机总体性能参数中的推力下降 $2.48 \%$, 耗油率增加 $2.54 \%$, 将会导致飞行成本增加; 发动机气 路性能中, 高压浴轮出口总温升高 $2.27 \%$, 高压浴轮出 口总压降低 $1.83 \%$, 高压浴轮总温的提高对发动机的 材料和结构提出更高的要求, 发动机的工作环境更加 恶劣. 为了维持发动机的性能, 减少蜕化造成的影响, 先进的发动机控制系统可以根据健康参数变化的实际 情况进行机载模型的修正，通过发动机的健康管理系 统和控制系统进行适当地调节, 力求使发动机工作在 最优或次优状态. 可见故障检测技术有助于发动机的 性能维护, 对于航空发动机的正常工作和性能提升方 面具有重要意义.

\section{3 自适应神经网络算法设计}

\section{1 常规神经网络算法}

人工神经网络是一种通过对大脑神经网络的结构 
和功能模拟而建立起来的信息处理系统. 它可以被认 为是一种具有大规模并行计算和学习能力的数学模 型，可以用来逼近任何难以用数学语言或者规则描述 的非线性系统. 针对航空发动机大时变、大包线、强 非线性和不确定性的特点，神经网络非常适合应用于 航空发动机的研发中.

前馈型神经网络的各神经元从输入层开始，接收 前一级输入, 并输入到下一级, 直至输出层, 整个网络 中无反馈. 它通常包含一个或多个隐含层, 前馈网络算 法过程如图2所示, 计算步骤如下 ${ }^{[5]}$.

(1) 设定网络结构, 进行初始化

给定输入矢量 $\boldsymbol{X}=\left(x_{1}, x_{2}, \cdots, x_{n}\right)$, 期望的输出矢 量 $\boldsymbol{Y}=\left(y_{1}, y_{2}, \cdots, y_{m}\right)$ 和误差要求. 对所有的权值赋以较 小的随机数, 并对阈值设定初值.

(2) 前向传播

计算神经元实际的输出:

隐含层第 $i$ 个神经元的输出为

$y 1_{i}=f 1\left(\sum_{j=1}^{m} w 1_{i, j} x_{j}+b 1_{i}\right), i=1,2, \cdots m$.

输出层第 $k$ 个神经元的输出为

$$
y 2_{k}=f 2\left(\sum_{i=1}^{n} w 2_{k, i} y 1_{i}+b 2_{k}\right), k=1,2, \cdots n .
$$

计算误差. 误差 $e_{k}=y_{k}-y 2_{k}$, 定义误差函数为

$E=\frac{1}{2} \sum_{k=1}^{l}\left(y_{k}-y 2_{k}\right)^{2}$.

(3) 梯度下降法求解网络权值变化

令 $\xi$ 为梯度下降的比例系数, 用来表示网络的学习 速率, 输出层阈值的变化为

$$
\begin{aligned}
\Delta b 2_{k, i} & =-\xi \frac{\partial E}{\partial b 2_{k, i}} \\
& =-\xi \frac{\partial E}{\partial y 2_{k}} \frac{\partial y 2_{k}}{\partial b 2_{k, i}} \\
& =\xi\left(y_{k}-y 2_{k}\right) f 2^{\prime} .
\end{aligned}
$$

输入层权值的变化为

$$
\begin{aligned}
\Delta w 2_{k, i} & =-\xi \frac{\partial E}{\partial w 2_{k, i}} \\
& =-\xi \frac{\partial E}{\partial y 2_{k}} \frac{\partial y 2_{k}}{\partial w 2_{k, i}} \\
& =\xi\left(y_{k}-y 2_{k}\right) f 2^{\prime} y 1_{i} .
\end{aligned}
$$

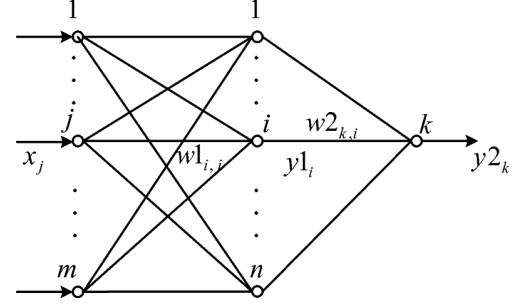

图 2 单隐含层前馈网络结构示意图

隐含层阈值的变化为

$$
\begin{aligned}
\Delta b 1_{i, j} & =-\xi \frac{\partial E}{\partial b 1_{i, j}}=-\xi \frac{\partial E}{\partial y 2_{k}} \frac{\partial y 2_{k}}{\partial y 1_{i}} \frac{\partial y 1_{i}}{\partial b 1_{i, j}} \\
& =\xi \sum_{k=1}^{l}\left(y_{k}-y 2_{k}\right) f 2^{\prime} w 2_{k, j} f 1 .
\end{aligned}
$$

输入层权值的变化为

$$
\begin{aligned}
\Delta w 1_{i, j} & =-\xi \frac{\partial E}{\partial w 1_{i, j}}=-\xi \frac{\partial E}{\partial y 2_{k}} \frac{\partial y 2_{k}}{\partial y 1_{i}} \frac{\partial y 1_{i}}{\partial w 1_{i, j}} \\
& =\xi \sum_{k=1}^{l}\left(y_{k}-y 2_{k}\right) f 2^{\prime} w 2_{k, j} f 1^{\prime} x_{j} .
\end{aligned}
$$

BP(back propagation)神经网络是前馈型神经网络 的一种典型形式. Hecht-Nielsen ${ }^{[6]}$ 从理论上证明了一个 隐含层为 3 层的 $\mathrm{BP}$ 神经网络, 能够有效逼近任意的连 续函数. 它有许多优点，例如善于处理大量样本、便 于信息处理、可以进行函数拟合或插值等，因此被广 泛应用于函数逼近、分类处理、参数 (状态) 识别和数 据压缩等．在神经网络的实际应用中， $80 \%$ $90 \%$ 都是 采用BP神经网络或其变形.

BP神经网络的基本思想是最小二乘法计算误差 逆传播, 采用每个权值变化时误差的导数, 反向调整权 值, 以此减小实际输出和期望输出之间的误差. 它除了 前向传播外, 还需要计算反向传播. 各层神经元的信息 只受前一层神经元的影响, 如果输出层的期望值误差 满足给定要求, 则完成学习; 否则进入反向传播, 将误 差信号返回，重新修改神经元的权值，使网络误差减 小. 反推误差的过程如下:

1) 计算输出层误差

$e_{k}=y_{k}-y 2_{k}$

2) 通过输出层激活函数导数, 求解系数

$\delta_{k, i}=e_{k} f 2^{\prime}$;

3) 计算隐含层权值变化量 
$\Delta w 2_{k, i}=\xi \delta_{k, i} y 1_{i}$,

并且计算

$e_{k} \sum_{k=1}^{l}\left(y_{k, i}-y 2_{k, i}\right) ;$

4) 通过隐含层激活函数导数, 求解系数

$\delta_{i, j}=e_{i} f 1^{\prime} ;$

5) 计算输入层权值变化量 $\Delta w 1_{i, j}$ :

$\Delta w 1_{k, i}=\xi \sum_{k=1}^{l}\left(y_{k}-y 2_{k}\right) f 2^{\prime} w 2_{k, i} f 1^{\prime} x_{j}=\xi \delta_{i, j} x_{j}$.

如果是多隐含层的网络, 则反复计算步骤 4 和 5 , 直 至计算到输入层为止. 这个误差反向传播的模式如图 3 所示.

\section{2 自适应神经网络算法}

常规的BP神经网络存在一定缺陷，首先基于梯度 法的网络误差计算模式决定了梯度下降不能过快，否 则容易引起网络计算不稳定，这导致网络收玫速度受 到了限制; 其次学习速率难以选择，初始学习速率太 大或太小都会影响网络的性能; 此外, 网络训练过程 中容易陷入局部最小，一般只能通过改变初始值和网 络参数, 经过反复尝试获取全局最优或次优解.

针对这些缺陷, 考虑发动机模型的特点, 在前馈型 (BP)神经网络的基础上，融合前人对于 $\mathrm{BP}$ 神经网络的 改进方法 ${ }^{[7,8]}$, 以及文献 $[9,10]$ 中的动量BP算法(momentum back propagation algorithm, MBPA), 设计了一种具 有自适应能力的神经网络.

对于式(8), 从第 $k$ 步学习到第 $k+1$ 步学习的过程 中, 反向传播的权值调整公式为

$w_{i, j}(k+1)=w_{i, j}(k)+\Delta w_{i, j}(k)$.

\section{变化量为}

$\Delta w_{i, j}(k)=-\xi \frac{\partial E(k)}{\partial w_{i, j}(k)}=-\xi w_{i, j}\left[b_{i, j}(k), w_{i, j}(k)\right]$,

式中, $E(k)$ 是网络的总加权误差, $b(k)$ 和 $w(k)$ 是按照式 (7)和(8)求得的关于阈值和权值的函数.

根据式(10), 固定的 $\xi$ 容易造成神经网络学习时效 率不高, 因为神经网络基于负梯度下降的思想, 初始时 刻希望采用大的 $\xi$, 使网络误差迅速下降, 加快学习速 率; 而接近收玫时, 又希望 $\xi$ 减小, 使网络能够减缓下降

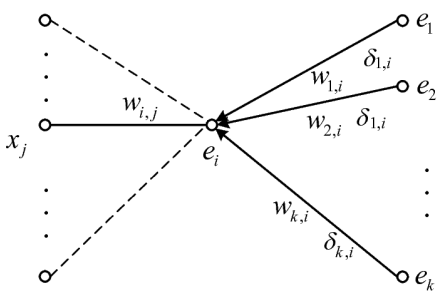

图 3 误差反向传播示意图

速度, 避免过调, 只在局部误差曲面加以微调即可. 因 此, 设定一种算法, 通过引入比例因子 $\xi_{1} \in(0,1]$ 调整学 习速率 $\xi$ 的大小; 通过引入门限值 $\xi_{2}$, 限定 $\xi_{1}$ 的其作用范 围, 进而对 $\xi$ 值加以修正:

$\xi=\left\{\begin{array}{l}\frac{1}{\xi_{1}} \xi, \frac{E(k+1)}{E(k)}<\xi_{2}, \\ \xi_{1} \xi, \frac{E(k+1)}{E(k)}>\xi_{2} .\end{array}\right.$

以 $\xi_{2}=1$ 为例, 当下一步误差 $E(k+1)$ 小于上一步误 差 $E(k)$, 总误差下降, 说明网络学习的方向正确, 通过 $\xi$ 除以小于 1 的 $\xi_{1}$ 来放大学习速率, 加快学习步伐; 当下 一步误差 $E(k+1)$ 大于上一步误差 $E(k)$, 总误差增加, 说明网络学习方向错误, 对样本的学习过度, 通过 $\xi$ 乘 以小于 1 的 $\xi_{1}$ 来减缓学习速率, 及时修正学习方向. 这 样的算法既可以提高算法的收玫速度, 又可以维持算 法稳定, 保证算法收玫于极小值.

此外, 为了避免网络可能陷入浅的局部极小值, 利 用增加一个“动量因子”的调整方法，使网络在收玫过 程中, 利用“惯性”作用, 可能滑过这些极小值. 具体来 说, 在反向传播的基础上, 将当前权值变化量与上一步 权值变化量进行加权求和, 作为新的当前权值变化 量. 采用动量因子 $m_{c} \in[0,1]$, 相应地, 权值调节公式 写为

$\Delta w_{i, j}(k+1)=\left(1-m_{c}\right) \xi \delta_{i}(k) x_{j}(k)+m_{c} \Delta w_{i, j}(k)$,

$\Delta b_{i}(k+1)=\left(1-m_{c}\right) \xi \delta_{i}(k)+m_{c} \Delta b_{i}(k)$.

当 $m_{c}=0$ 时, 方程退化为 $\mathrm{BP}$ 神经网络的基本算法, 当 $m_{c}=1$, 当前权值与上一步相同, 网络学习停滞. BP 神经网络本身在权值进入误差曲面底部“平坦”区域 后, 由于 $\delta_{i} \rightarrow 0 \Delta w_{i, j}(k+1) \rightarrow \Delta w_{i, j}(k)$, 导致 $\Delta w_{i, j}(k) \rightarrow 0$, 网络进入平滑收玫的状态. 如果该极小值只是局部极 
小值而非全局极小值，则网络难以跳出此局部极小值. 增加了动量因子 $m_{c}$ 后, 通过调节, $m_{c}$ 有助于防 止 $\Delta w_{i, j}(k) \rightarrow 0$, 使网络能够从局部极小值跳出. 增加 了动量因子 $m_{c}$ 后，可以使梯度方向的动量变化变得平 滑, 从而增加神经网络的稳定性.

为了防止修正后的权值过大或过小，按照以下方 式调节 $m_{c}$ :

$m_{c}= \begin{cases}0, & E(k+1)>1.05 E(k), \\ 0.95, & E(k+1)<E(k), \\ m_{c}, & \text { else. }\end{cases}$

综合式(9)和(12),

$$
\begin{aligned}
w_{i, j}(k+1)= & w_{i, j}(k)-\xi w_{i, j}\left[b_{i, j}(k), w_{i, j}(k)\right] \\
& +\left(1-m_{c}\right) \xi \delta_{i}(k) x_{j}(k)+m_{c} \Delta w_{i, j}(k) .
\end{aligned}
$$

按照此方法计算输出层和隐含层的权值变化，神 经网络的性能得到了一定的改进, 学习速率增加, 避 免了陷入局部极小值，网络的拟合和泛化能力得到了 一定的提高.

\section{4 基于Pearson相关系数的相关分析算法}

\section{1 航空发动机参数的相关分析}

研究发现，发动机非线性部件级模型的输入参数 和输出参数之间存在一定的相关关系: 有的输入参数 对部分输出参数影响较大, 而有的参数影响较小. 研 究中通过相关分析的方法，对这些参数之间的相关性 作进一步研究.

相关分析(correlation analysis), 是研究随机变量的 一种统计方法, 用来研究变量之间是否存在特殊的依 存关系，并且对于有相互依存关系的变量，探讨其相 关的程度. 常用的相关分析方法，主要包括基于统计 理论的相关分析、基于互信息的相关分析、基于矩阵 计算的相关分析和基于距离的相关分析. 针对本文研 究的发动机模型及神经网络采样需求的特点, 选用了 基于统计理论的相关分析中的Pearson相关分析(Pearson correlation coefficient, PCC)的方法.

\subsection{Pearson相关系数}

PCC用来衡量两个变量之间的相关关系，也被称 为Pearson积矩相关系数 (Pearson product-moment correlation coefficient, PPMCC), 它由Pearson ${ }^{[11]}$ 提出, 并由
Gain和Fisher完善了计算方法. 对于两个变量序列 $\boldsymbol{X}$ 和 $\boldsymbol{Y}, \mathrm{PCC}$ 的计算方法如下:

$\rho_{X, Y}=\frac{\operatorname{cov}(\boldsymbol{X}, \boldsymbol{Y})}{\sigma_{X} \sigma_{Y}}=\frac{E\left[\left(\boldsymbol{X}-\mu_{X}\right)\left(\boldsymbol{Y}-\mu_{Y}\right)\right]}{\sigma_{X} \sigma_{Y}}$,

其中, $\sigma_{X}$ 和 $\sigma_{Y}$ 分别表示 $\boldsymbol{X}$ 和 $\boldsymbol{Y}$ 的标准差, $\operatorname{cov}(\boldsymbol{X}, \boldsymbol{Y})$ 表 示 $\boldsymbol{X}$ 和 $\boldsymbol{Y}$ 的协方差. $\mu_{X}$ 和 $\mu_{Y}$ 分别表示 $\boldsymbol{X}$ 和 $\boldsymbol{Y}$ 的均值. $\rho_{X, Y} \in[-1,1]$, 一般情况下, $\rho_{X, Y}$ 对相关关系的描述, 满 足以下原则:

$\left|\rho_{X, Y}\right| \in[0.8,1]$, 极强相关;

$\left|\rho_{X, Y}\right| \in[0.6,0.8)$, 强相关;

$\rho_{X, Y} \mid \in[0.4,0.6)$, 中等程度相关;

$\left|\rho_{X, Y}\right| \in[0.2,0.4)$, 弱相关;

$\left|\rho_{X, Y}\right| \in[0,0.2)$, 极弱相关或无相关.

对发动机部件级模型计算 $N$ 个工况的样本，并将 需要分析的发动机 $M$ 个参数组成样本数据矩阵:

$\left(\begin{array}{lll}x_{11} & \ldots & x_{1 N} \\ \vdots & \ddots & \vdots \\ x_{M 1} & \cdots & x_{M N}\end{array}\right)$.

令 $\boldsymbol{x}_{i} \in R^{1 \times N}$ 表示其中的第 $i$ 行, $i=1,2, \cdots M$, 表示 所有样本中的第 $i$ 个参数序列; $\boldsymbol{x}_{j} \in R^{1 \times N}$ 表示第 $j$ 个参 数序列, $j=1,2, \cdots M$.

$$
\begin{aligned}
\operatorname{cov}\left(\boldsymbol{x}_{i}, \boldsymbol{x}_{j}\right) & =E\left[\left(\boldsymbol{x}_{i}-\bar{x}_{i}\right)\left(\boldsymbol{x}_{j}-\bar{x}_{j}\right)\right] \\
& =E\left(\boldsymbol{x}_{i} \boldsymbol{x}_{j}\right)-2 E\left(\boldsymbol{x}_{i}\right) E\left(\boldsymbol{x}_{j}\right)+E\left(\boldsymbol{x}_{i}\right) E\left(\boldsymbol{x}_{j}\right) \\
& =E\left(\boldsymbol{x}_{i} \boldsymbol{x}_{j}\right)-E\left(\boldsymbol{x}_{i}\right) E\left(\boldsymbol{x}_{j}\right),
\end{aligned}
$$

其中, $\bar{x}_{i}=E\left(\boldsymbol{x}_{i}\right), \bar{x}_{j}=E\left(\boldsymbol{x}_{j}\right)$.

相应的 $\mathrm{PCC}$ 通过下式计算:

$\rho_{i, j}=\frac{\operatorname{cov}\left(\boldsymbol{x}_{i}, \boldsymbol{x}_{j}\right)}{\sqrt{\operatorname{cov}\left(\boldsymbol{x}_{i}, \boldsymbol{x}_{i}\right)} \sqrt{\operatorname{cov}\left(\boldsymbol{x}_{j}, \boldsymbol{x}_{j}\right)}}$.

将相关系数组成相关系数矩阵 $\boldsymbol{R}_{\rho} \in R^{M \times M}$ :

$\mathbf{R}_{\rho}=\left(\begin{array}{lll}\rho_{1,1} & \cdots & \rho_{1, M} \\ \vdots & \ddots & \vdots \\ \rho_{M, 1} & \cdots & \rho_{M, M}\end{array}\right)$.

$\boldsymbol{R}_{\rho}$ 的每个元素用来表征所在行与列的下标编号 所对应的两个发动机参数的相关性. 


\section{5 发动机故障诊断算法验证}

\section{1 发动机特征参数提取}

本文在研究时, 从部件级模型计算得到的样本中 提取以下 16 个参数, 分析其中的相关性:

$\left[\eta_{\text {fan }}, \eta_{\mathrm{HP}}, \eta_{\mathrm{HT}}, \eta_{\mathrm{LT}}, f_{0}, F_{\mathrm{s}}, F N, \mathrm{sfc}\right.$,
$\left.\quad P_{t 25}, P_{t 3}, P_{t 45}, P_{t 5}, T_{t 25}, T_{t 3}, T_{t 45}, T_{t 5}\right]$.

这些参数为: $\eta_{\mathrm{fan}}$, 风扇效率系数; $\eta_{\mathrm{HP}}$, 高压压气机 效率系数; $\eta_{\mathrm{HT}}$, 高压浴轮效率系数; $\eta_{\mathrm{LT}}$, 低压浴轮效率 系数; $f_{0}$, 油气比; $F_{\mathrm{s}}$, 单位推力; $F N$, 推力; $\mathrm{sfc}$, 耗油率; $P_{t 25}$, 高压压气机进口总压; $P_{t 3}$, 高压压气机出口总压; $P_{t 45}$, 高压涡轮出口总压; $P_{t 5}$, 低压浴轮出口总压; $T_{t 25}$, 高 压压气机进口总温; $T_{t 3}$, 高压压气机出口总温; $T_{t 45}$, 高 压浴轮出口总温; $T_{t 5}$, 低压浴轮出口总温.

按照 4.2 节的方法, 将算得的 $\boldsymbol{R}_{\rho}$ 以矩阵图表示 (图4).

分析可见, 对于健康参数 $\left[\eta_{\mathrm{fan}}, \eta_{\mathrm{HP}}, \eta_{\mathrm{HT}}, \eta_{\mathrm{LT}}\right.$ 来说, 与 其相关的气路参数序列集中在 5 个参数: 高压涡轮出口 总压 $P_{t 4}$, 低压浴轮出口总压 $P_{t 5}$, 高压压气机出口总 温 $T_{t 3}$, 高压浴轮出口总温 $T_{t 4}$, 低压浴轮出口总温 $T_{t 5}$; 而 其他 3 个气路参数与之相关性不大. 因此, 提取每个样 本中的这些特定参数作为神经网络的输入参数和输出 参数, 用于训练神经网络. 最终所取的神经网络的输入 和输出分别记为

$$
\left\{\begin{array}{l}
\boldsymbol{x}=\left[\begin{array}{lllll}
P_{t 4} & P_{t 5} & T_{t 3} & T_{t 4} & T_{t 5}
\end{array}\right]^{\mathrm{T}}, \\
\boldsymbol{y}=\left[\begin{array}{llll}
\eta_{\text {fan }} & \eta_{\mathrm{HP}} & \eta_{\mathrm{HT}} & \eta_{\mathrm{LT}}
\end{array}\right]^{\mathrm{T}} .
\end{array}\right.
$$

\section{2 基于神经网络故障诊断算法验证}

首先根据一般的发动机非线性部件级模型，产生 发动机模型在各种故障情况发生蜕化的工况数据样本 库(随机选取其中 1037 个为神经网络的训练样本, 259 个为测试样本, 用来检验网络性能). 以 $\boldsymbol{P}_{\text {train }} \in R^{1037 \times 9}$ 表 示训练样本集, $\boldsymbol{P}_{\text {train,in }} \in R^{1037 \times 5}$ 表示训练样本输入, $\boldsymbol{P}_{\text {train,out }} \in R^{1037 \times 4}$ 表示训练样本输出. 同理, 以 $\boldsymbol{P}_{\text {test }} \in R^{259 \times 9}$ 表示测试样本集, $\boldsymbol{P}_{\text {test,in }} \in R^{259 \times 5}$ 表示测试 样本输入, $\boldsymbol{P}_{\text {test,out }} \in R^{259 \times 4}$ 表示测试样本输出.

将 $\boldsymbol{P}_{\text {train }}$ 用 BP神经网络训练得到输出 $\boldsymbol{y}_{\text {test }}$, 比较 $\boldsymbol{y}_{\text {test }}$

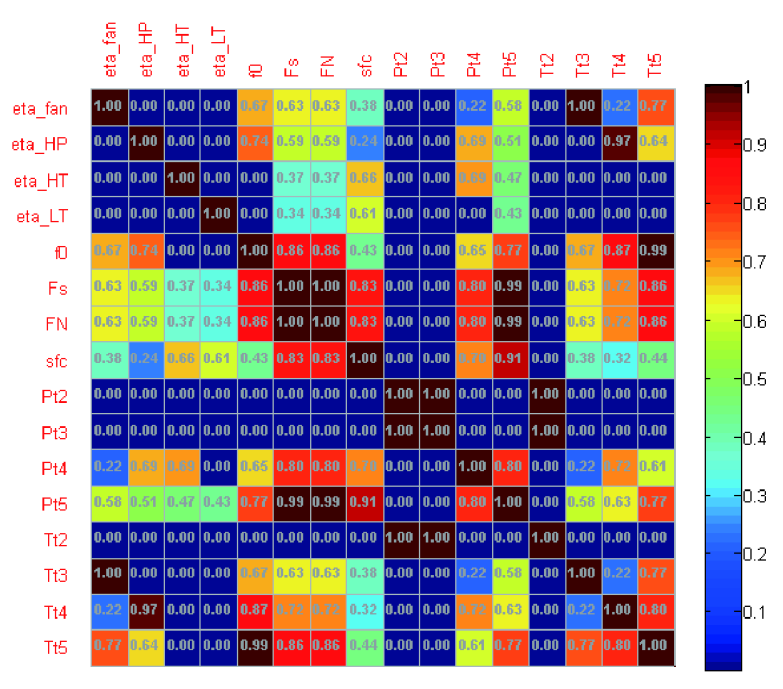

图 4 (网络版彩图)相关分析系数矩阵色块图

和 $\boldsymbol{P}_{\text {test,out. }}$ 由于有 259 个测试样本, 数量较多, 为了方便 观察结果, 画图时仅仅随机选取其中 50 个的数据结果 (图5). 图 5中, 横坐标为这 50 个数据按顺序的编号, 对 应的纵坐标中, “o”表示原来 $\boldsymbol{P}_{\text {test,out }}$ 的数据(用来代表发 动机真实健康参数), “*”表示神经网络估计的结果. 显 而易见，同一个样本的两个符号接近程度或重合度越 高, 说明神经网络对于该样本的健康参数估计越准确, 相应的故障诊断的精度越高.

图5结果表明, 无论是常规BP神经网络, 还是本文 提出的自适应神经网络, 这两种网络的收玫速率较快, 精度较高, 整体上对测试样本的估计比较准确. 对比通 过常规BP神经网络和自适应神经网络进行故障诊断 的结果, 可以发现, 后者的图中“*”与“o”的匹配度较高, 几乎所有的样本中, 两者的数据都是几乎重合的, 而前 者的图中“*”与“o”对应的数值明显有一定差异, 效果 远不如自适应神经网络的估计能力优异. 这一点从表 1 中的平均相对误差统计结果也可以发现. 这说明本 文设计的自适应神经网络，在精度上明显高于常规的 $\mathrm{BP}$ 神经网络, 更适合用于发动机健康参数的故障诊断.

图6表明, 常规BP神经网络在学习发动机的样本 时, 步长在反复增加、减小的过程中, 通过200步以上 的迭代依然未完全收玫. 而自适应神经网络则收玫较 快, 整体网络误差也小得多.

此外, 研究中发现, 常规的BP神经网络的计算效 果具有一定随机性, 浮动较大, 需要反复训练多次后, 

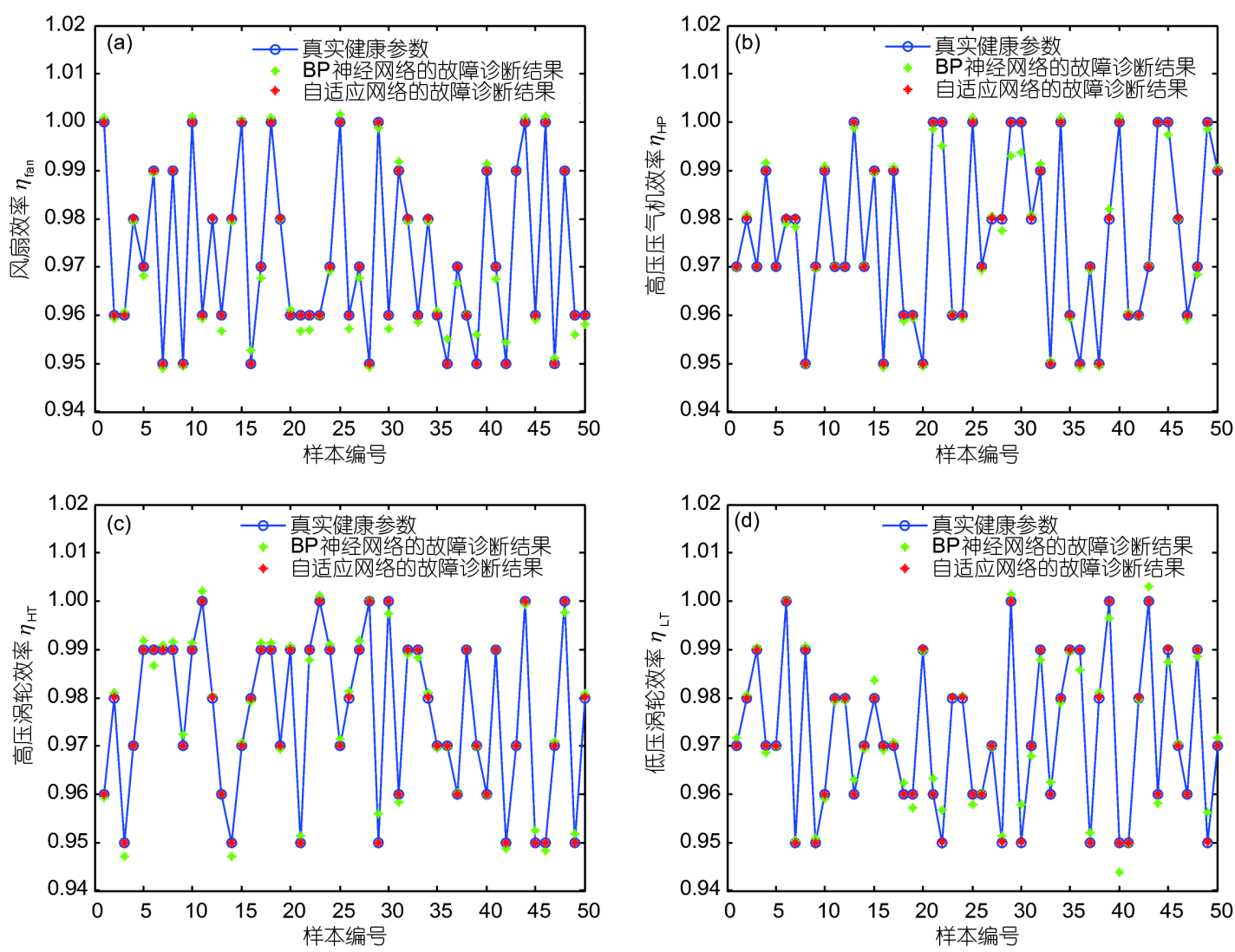

图 5 (网络版彩图)采用BP网络和自适应网络的故障诊断结果比较. (a) 风扇效率; (b) 高压压气机效率; (c) 高压浴轮效率; (d) 低压浴轮效率

(a)
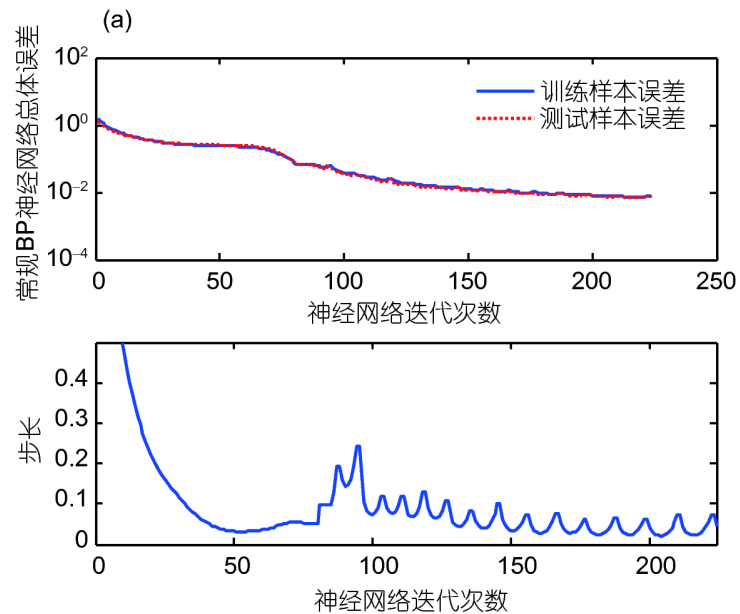

(b)
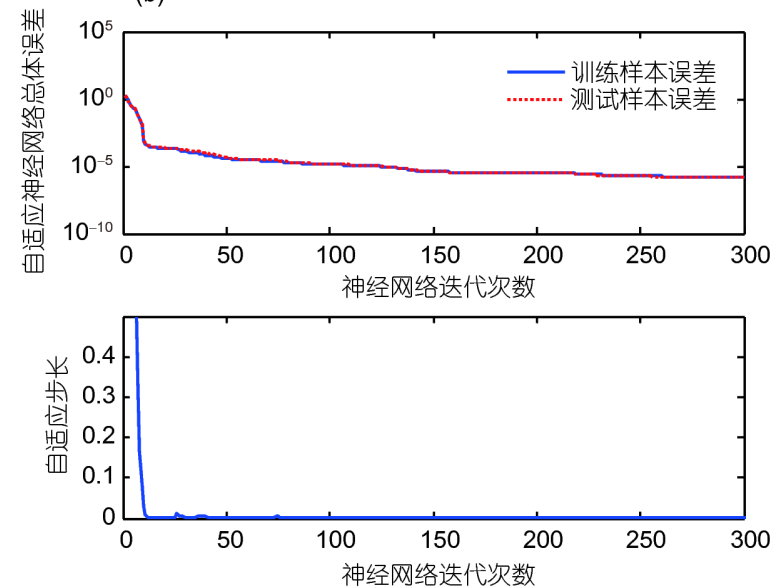

图 6 (网络版彩图)BP网络和自适应网络的迭代过程. (a) 常规BP神经网络; (b) 自适应神经网络

择优选取其中精度最高的网络，才能达到较为理想的 水平; 而自适应网络稳定性较高，几乎每次训练都能 收玫于全局最小值，保证训练得到的网络精度较高.
在工程上, 通常测试样本 $\boldsymbol{P}_{\text {test }}$ 中的 $\boldsymbol{P}_{\text {test,out }}$ 是具有相当大 的随机性的, 所以难以事先确定网络的置信度, 因此, 网络在学习过程中拥有这种稳定性也是非常重要的. 


\section{3 少量训练样本的故障诊断}

在5.2节的算例中，尽管网络较精确，但是一共用 到的样本数量达到了 1296 个, 其中训练样本也有 1000 个以上，实际工程中由于成本、时间等原因，无法通 过实验获取大量样本. 这要求在进行故障诊断时, 在 保证网络精确度达到一定要求的前提下，训练神经网
络的样本数量应当尽量减少. 显然, 样本数量过少会造 成神经网络学习效果降低. 因此, 研究中反复尝试了选 取少量训练样本, 降低 $\boldsymbol{P}_{\text {train }}$ 的维数. 最终, 采用本文提 出的自适应神经网络算法, 并且使用的训练样本数量 为 25 个时, 网络精度和样本数量达到一定的平衡.

作为对比，依然分别使用常规 $\mathrm{BP}$ 神经网络和本文 提出的自适应神经网络测试故障诊断的精度. 首先, 从

表 1 BP网络与自适应网络的平均相对误差比较

\begin{tabular}{ccccc}
\hline 健康参数 & $\eta_{\text {fan }}$ & $\eta_{\mathrm{HP}}$ & $\eta_{\mathrm{HT}}$ & $\eta_{\mathrm{LT}}$ \\
\hline BP神经网络 & $1.675 \times 10^{-3}$ & $1.086 \times 10^{-3}$ & $1.343 \times 10^{-3}$ & $2.377 \times 10^{-3}$ \\
自适应神经网络 & $1.295 \times 10^{-5}$ & $1.462 \times 10^{-5}$ & $2.680 \times 10^{-5}$ & $3.531 \times 10^{-5}$ \\
\hline
\end{tabular}

表 2 BP网络与自适应网络的平均相对误差比较(仅用 25 个训练样本)

\begin{tabular}{ccccc}
\hline 健康参数 & $\eta_{\text {fan }}$ & $\eta_{\mathrm{HP}}$ & $\eta_{\mathrm{HT}}$ & $\eta_{\mathrm{LT}}$ \\
\hline BP神经网络 & $6.371 \times 10^{-3}$ & $4.459 \times 10^{-3}$ & $1.261 \times 10^{-2}$ & $1.647 \times 10^{-2}$ \\
自适应神经网络 & $6.303 \times 10^{-4}$ & $1.727 \times 10^{-3}$ & $5.810 \times 10^{-4}$ & $8.444 \times 10^{-4}$ \\
\hline
\end{tabular}
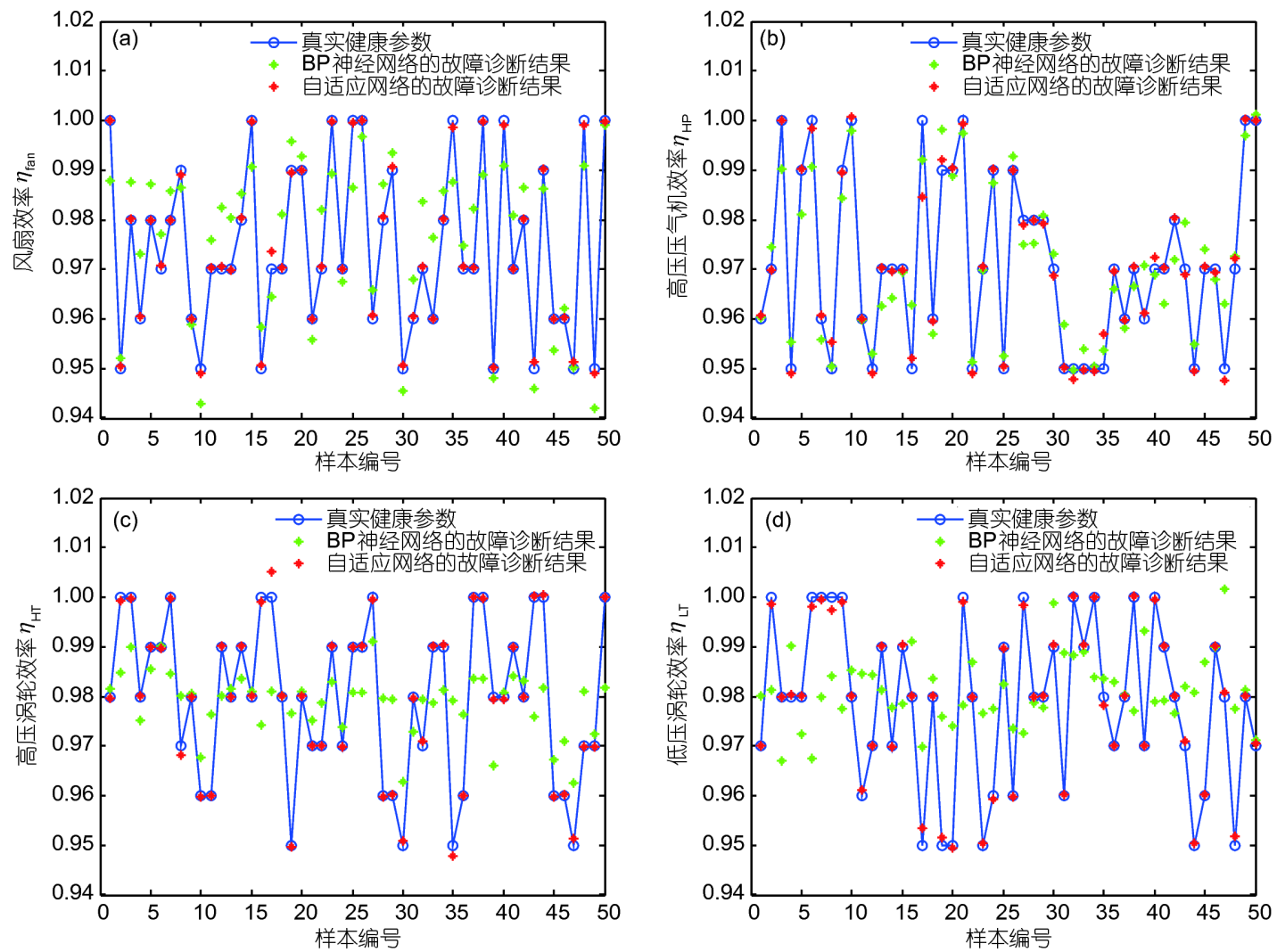

图 7 (网络版彩图)采用BP网络和自适应网络的故障诊断结果比较(仅用 25 个训练样本). (a) 风扇效率; (b) 高压压气机效率; (c) 高压浴轮效率; (d) 低压浴轮效率 

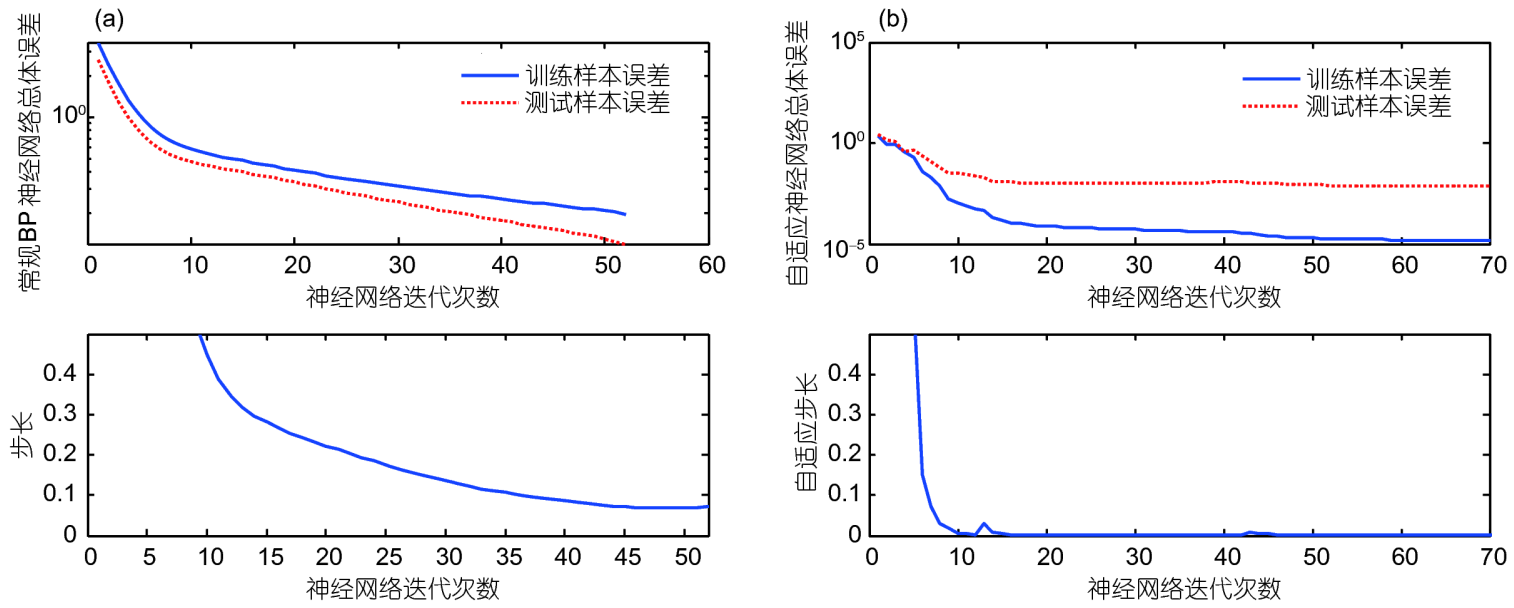

图 8 (网络版彩图)BP网络和自适应网络的迭代过程(仅用 25 个训练样本). (a) 常规BP神经网络; (b) 自适应神经网络

前述 1296 个样本库中, 随机选取相同的 25 个样本, 作为 训练样本, 分别训练两种神经网络; 然后从样本库随机 选取同样的 50 个样本, 用于测试两个训练好的网络的 性能.

计算此算例的常规BP神经网络与自适应网络的 平均相对误差, 统计在表2中.

从图7和表 2 中可见, 在训练样本数大幅度减少的 情况下, 常规BP神经网络的误差变得较大; 但是, 本文 提出的自适应网络对各个健康参数的估计整体误差较 小，相应地，诊断出的健康参数依然能保持较高的 精度.

常规BP神经网络的收敛过程如图8(a)所示，自适 应神经网络收敛过程如图8(b)所示. 图8可见，BP神经 网络收玫较慢, 误差也较大; 然而, 本文提出的自适应 网络收玫速度较快, 只需要 18 步左右即收玫. 本文提出 的自适应神经网络，其收玫性也要优于常规BP神经 网络.

\section{6 结论}

本文主要研究了通过神经网络进行发动机健康参
数故障诊断的方法，并设计了算例验证算法的有效性 和精度. 主要工作有以下几方面.

(1) 通过相关分析，合理选取影响发动机健康参 数的关键性能参数, 作为神经网络的输入和输入 参数.

(2) 为了弥补常规前馈型神经网络(BP神经网络) 的缺陷，设计了一种融合了比例因子和动量因子调控 的自适应神经网络，该网络稳定性好，不易陷入局部 最优.

（3）通过算例测试了神经网络的性能, 对比了基于 $\mathrm{BP}$ 神经网络和自适应网络的故障诊断结果的误差大 小，结果证明本文提出的自适应网络能够提高发动机 故障诊断的精度.

(4) 基于工程实用背景, 测试了在训练样本数较少 时算法的性能，结果证明该方法对于神经网络只有少 量样本时, 依然有理想的精度.

总之，本文设计的神经网络在航空发动机健康参 数的估计方面, 精度较高, 对于训练样本极少的情况, 也能够很好地训练和学习, 泛化能力强, 因此可以作为 一种针对发动机气路性能蜕化的故障诊断的参考 方法.

\section{参考文献}

1 藏军. 现代航空发动机控制技术. 北京: 航空工业出版社, 2016

2 Sellers J F, Daniele C J. DYNGEN: A program for calculating steady-state and transient performance of turbojet and turbofan engines. NASA Technical Report, 1975

3 Voloshchuk V. Calculation of gas turbine engine cycle. In: Rogalev N, ed. Thermal Engineering Studies with Excel, Mathcad and Internet. Cham: 
Springer International Publishing, 2016

4 廉䈗纯. 航空发动机原理. 西安: 西北工业大学出版社, 2005

5 张德丰. MATLAB神经网络编程. 北京: 化学工业出版社, 2011

6 Hecht-Nielsen R. Theory of the backpropagation neural network. In: Proceedings of the International Joint Conference on Neural Networks. Washington DC, 1989

7 Hua H Y, Zhong X Z. A self-adaptive learning algorithm for BP network. Syst Eng-Theory Pract, 2000, 20: 93-98

8 Anderson N J, Czapla-Myers J S. Identification of the proportion of the dynamic load in generalized load based on adaptive BP network. Trans China Electrotech Soc, 2013, 28: 78-83

9 Wu W, Zhang N M, Li Z X, et al. Convergence of gradient method with momentum for back-propagation neural networks. J Comput Math, 2008, 26: $613-623$

10 Shao H, Zheng G. Convergence analysis of a back-propagation algorithm with adaptive momentum. Neurocomputing, 2011, 74: 749-752

11 Adler J, Parmryd I. Quantifying colocalization by correlation: The Pearson correlation coefficient is superior to the Mander's overlap coefficient. Cytometry, 2010, 77A: 733-742

\title{
Fault diagnosis of aero-engine based on self-adaptive neural network
}

\author{
AI JianLiang $^{1} \&$ YANG XiZhong ${ }^{1,2}$ \\ ${ }^{1}$ Department of Aeronautics and Astronautics, Fudan University, Shanghai 200433, China; \\ ${ }^{2}$ Chinese Aeronautical Radio Electronics Research Institute, Shanghai 200233, China
}

As the gas path components' performance cannot avoid degeneration while the aero-engine is working, the related fault diagnosis technology is of great meaning to the aero-engine's health management system. Aiming at the aero-engine's nonlinear model at the design point, this paper optimizes the selection rules of the neural network's input parameters and output parameters through relative analysis of PCC (Pearson correlation coefficient). For the regular BP (back propagation) neural network's weakness of an unstable convergence speed and easy to fall into minimum value, this paper designs a new method via self-adaptive neural network that can accurately estimate the degeneration level of the aero-engine. This method combines scale factor and momentum factor, improves the learning speed of network and enhances the confidence level of neural network as well as the generalization ability of engine's model parameters. The results prove that the self-adaptive neural network method proposed in this paper has better accuracy than the regular BP neural network, and when the training samples are not too many, the training can also produce an ideal network, which guarantees a good accuracy for the fault diagnosis of the aero-engine's health parameters.

\section{aero-engine, health parameters, fault diagnosis, self-adaptive neural network, correlation analysis}

doi: $10.1360 / \mathrm{N} 092017-00224$ 